\title{
Diagnostic associations in a large and consecutively identified population positive for anti-SSA and/or anti-SSB: the range of associated diseases differs according to the detailed serotype
}

\author{
I Peene, L Meheus, E M Veys, F De Keyser
}

Ann Rheum Dis 2002;61:1090-1094

See end of article for authors' affiliations

Correspondence to Dr I Peene, Department of Rheumatology 0K12IB, De Pintelaan 185, 9000 Gent Belgium;

filip.dekeyser@rug.ac.be

Accepted 7 May 2002
Objective: To determine the diagnostic distribution in a consecutive anti-SSA and/or anti-SSB positive population.

Methods: A total of 15937 serum samples from 10550 consecutive patients were analysed for antinuclear antibodies (ANAs) on HEp-2 cells. Serum samples positive for ANAs were analysed by immunodiffusion and line immunoassay with recombinant SSA-Ro52, natural SSA-Ro60, and recombinant SSB. Results: Among ANA positive patients in whom clinical information was available, 181 consecutive patients with anti-SSA and/or anti-SSB antibodies were identified, Disease associations were systemic lupus erythematosus (SLE) $(45.3 \%)$, primary Sïorgen's syndrome (pSS) $(14.4 \%)$, scleroderma $(8.8 \%)$, RA $(7.7 \%)$, cutaneous lupus $(7.7 \%)$, and dermatomyositis $(2.2 \%)$. The ratio of diagnoses differed according to the anti-SSA/anti-SSB serotype. Scleroderma and dermatomyositis were enriched among mono-Ro52 reactive serum samples $(34.2 \%$ and $10.5 \%$ respectively). Single reactivity towards Ro60 or anti-Ro60 with anti-Ro52 predisposed for SLE $(80.0 \%$ and $52.2 \%$ respectively). Triple reactivity towards Ro52, Ro60, and SSB was primarily linked with SLE (55.8\%) followed by pSS (20.9\%). AntiSSA on immunodiffusion increased the chance for SLE $(62.8 \%)$, whereas isolated anti-SSB reactivity on immunodiffusion was less indicative for SLE (14.3\%) and predisposed more for cutaneous lupus $(23.8 \%)$ and pSS (33.3\%).

Conclusion: The diagnostic range associated with anti-SSA or anti-SSB reactivity differs significantly according to the detailed serotype defined by line immunoassay and immunodiffusion.
A nti-SSA autoantibodies are the most often identified antinuclear antibodies (ANAs). ${ }^{1}$ They were initially recognised in systemic lupus erythematosus (SLE) and Sjögren's syndrome (SS). It soon became evident that anti-SSA antibodies were also present in serum samples from patients with other autoimmune diseases. ${ }^{2-4}$

Anti-SSA antibodies may react against at least two different protein determinants: a $52 \mathrm{kD}$ protein (Ro52) and a $60 \mathrm{kD}$ protein (Ro60). The Ro60 molecule and the SSB protein have been shown to be components of the same ribonucleoprotein complex. It remains uncertain, however, whether the Ro52 protein is also a component of this complex. Evidence has been presented that Ro52 and Ro60 are structurally unrelated and, if they are associated on the molecular level, that this association is most likely transitory. ${ }^{5-8}$

The anti-SSA response may be directed towards either of these components. Anti-SSB antibodies often occur together with anti-SSA antibodies. Therefore, we consider anti-Ro52, anti-Ro60, and anti-SSB reactivity together.

Various serological assays exist for the detection of anti-SSA and anti-SSB reactivity. Enzyme linked immunosorbent assays (ELISAs), double immunodiffusion, and counterimmunoelectrophoresis techniques are the commonly used tests in clinical laboratories. Recently, recombinant Ro52 and recombinant or native Ro60 have been introduced in different assays to detect autoantibodies towards one or both components. $^{9-11}$ The diagnostic value of these subsets of antibodies is, however, not clear. We describe here the diagnostic association of anti-SSA and anti-SSB antibodies identified in a large consecutive population, from whom serum samples were referred to the laboratory for ANA testing.

\section{PATIENTS AND METHODS Patients}

A total of 15937 serum samples from 10550 consecutive patients were referred to the rheumatology laboratory (Ghent University Hospital) over a three year period (1996-9) for ANA detection and identification. These samples were referred by in house rheumatologists (25\% of the samples), internal medicine specialists (15\%), gastroenterologists $(7 \%)$, dermatologists $(5 \%)$, neurologists $(5 \%)$, nephrologists $(3 \%)$, and external hospitals or laboratories (23\%).

Serum samples positive for ANA were further analysed in parallel by double immunodiffusion with thymus/spleen nuclear extract (mammalian extracted nuclear antigen, Immunoconcepts, Sacramento, CA, USA) and by line immunoassay coated with nuclear antigens, including recombinant Ro52 and SSB, and natural Ro60 (INNO LIA ANA K1090, Innogenetics, Gent, Belgium). For each patient showing antiSSA (Ro52 and/or Ro60) and/or anti-SSB reactivity, clinical information was asked from the doctor who had ordered the test. Thus, diagnostic information could be obtained in 181 patients. Patients who were classified as having SLE, rheumatoid arthritis (RA), scleroderma ( $\mathrm{Scl}$ ), primary SS (pSS), or dermatomyositis (DM) met the classification criteria for the

Abbreviations: ANA, antinuclear antibodies; $B C I P$,

5-bromo-4-chloro-3-indolyl phosphatase; CLE, cutaneous lupus erythematosus; DM, dermatomyositis; ELISA, enzyme linked immunosorbent assay; pSS, primary Siögren's syndrome; RA, rheumatoid arthritis; Ro52, 52 kD protein; Ro60, 60 kD protein; Scl, scleroderma; SLE, systemic lupus erythematosus; SS, Sjögren's syndrome 

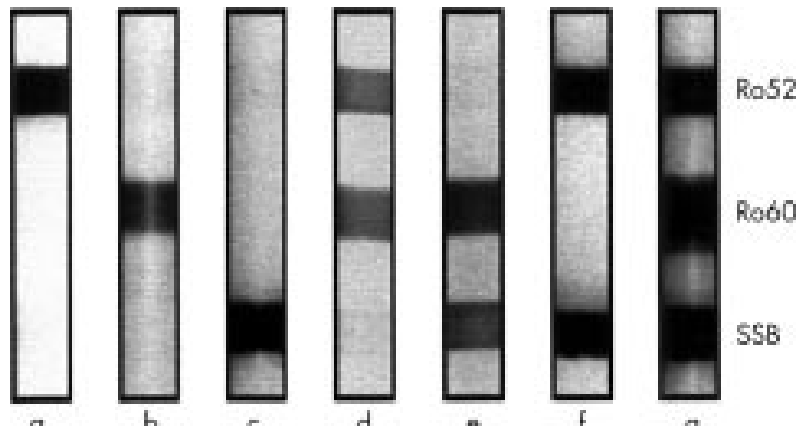

Figure 1 Different combinations of reactivities towards Ro52, Ro60, and SSB defined by line immunoassay. Lane a: anti-Ro52 antibodies; lane b: anti-Ro60 antibodies; lane c: anti-SSB antibodies; lane d: anti-Ro52 and anti-Ro60 antibodies; lane e: anti-Ro60 and anti-SSB antibodies; lane f: anti-Ro52 and anti-SSB antibodies; lane g: anti-Ro52 and anti-Ro60 and anti-SSB antibodies.

respective diseases. ${ }^{12-16}$ Patients classified with cutaneous lupus erythematosus (CLE) had CLE established by biopsy but did not meet the criteria for SLE.

\section{Indirect immunofluorescence on HEp-2 cells}

Serum diluted 1:40 in phosphate buffered saline (PBS) was overlaid onto fixed HEp-2 cells (Medica inc, Carlsbad, CA, USA) for 30 minutes at room temperature. Slides were washed twice for five minutes each with PBS, overlaid with fluoresceinated total immunoglobulin, and incubated for an additional 30 minutes. After washing twice, a coverslip was placed over the slide, and the slides were read using a fluorescence microscope at $40 \times$ power.

\section{Double immunodiffusion}

Precipitating antibodies against extractable nuclear antigens were detected by double immunodiffusion on Ouchterlony plates with thymus/spleen nuclear extract (mammalian extracted nuclear antigen, Immunoconcepts, Sacramento, CA, USA). Antibody specificity was determined by comparison with a reference serum.

\section{Line immunoassay}

A line immunoassay coated with nuclear antigens, including full size Escherichia coli derived recombinant Ro52, recombinant SSB, and natural Ro60 (INNO-LIA ANA K1090), was used. The test was performed according to the manufacturer's instructions. Briefly, the nylon strips were incubated with serum at a 1:200 dilution. A goat antihuman IgG labelled with alkaline phosphatase was allowed to bind to the antigenantibody complex. The enzyme substrate and chromogen 5-bromo-4-chloro-3-indolyl phosphatase (BCIP) produces a dark brown colour in proportion to the amount of specific autoantibody in the test sample. Sulphuric acid stops the colour development (fig 1).

\section{Statistics}

Percentages and their corresponding 95\% confidence intervals (95\% CIs) (one binomial) and Fisher's exact test were performed by StatXact.

\section{RESULTS}

Testing for ANA consecutively performed on 15937 serum samples from 10550 patients referred to our laboratory over a three year period, was positive in 4691 samples from 2669 patients. Anti-SSA and/or anti-SSB reactivity was found in $11.8 \%$ of ANA positive serum samples. ${ }^{1}$ We identified 181 consecutive patients with anti-SSA and/or anti-SSB antibodies

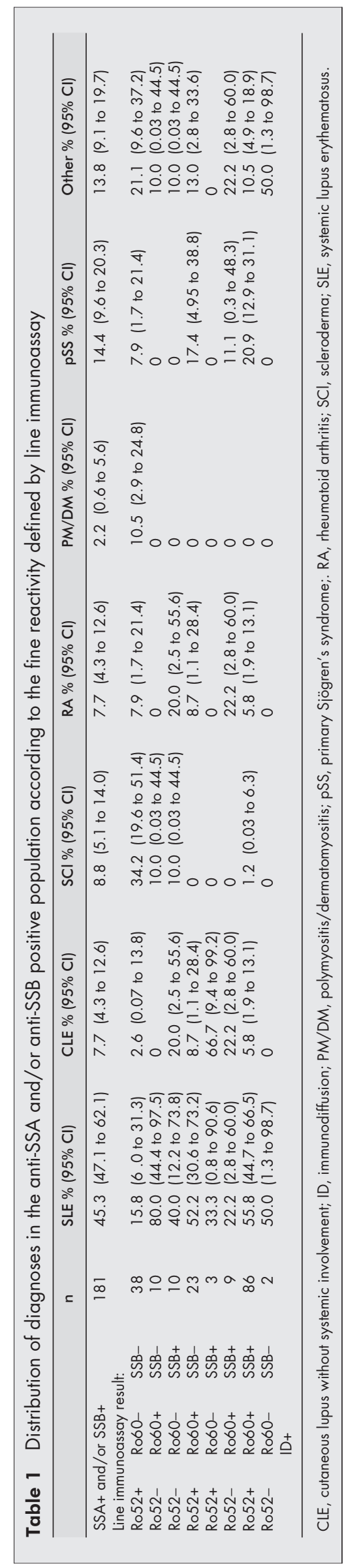



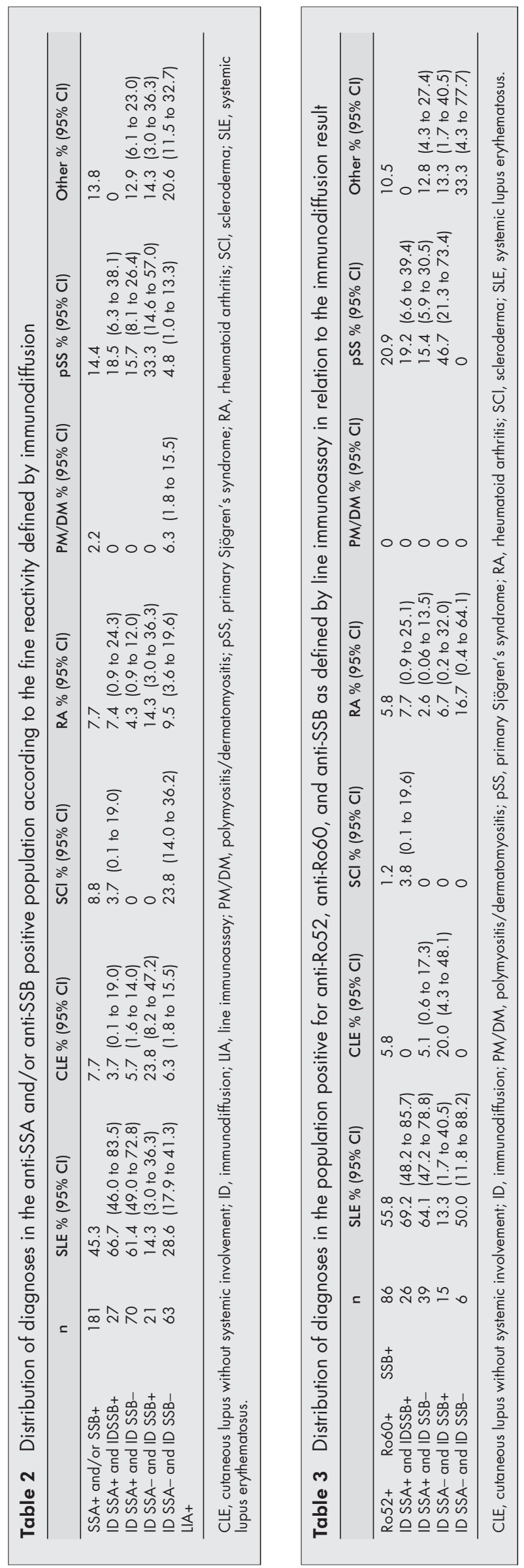

among those patients in whom clinical information was available to us. In patients in whom multiple serum samples were tested over time, anti-SSA/anti-SSB status remained largely constant.

Tables 1 and 2 give the distribution of associated diseases. The most prevalent diagnosis in the entire anti-SSA (antiRo52 and/or anti-Ro60) and/or anti-SSB positive population was lupus (53.0\%; SLE $(n=82)$ and cutaneous LE $(n=14))$. Other diagnoses included pSS $(n=26), R A(n=14)$, systemic sclerosis $(n=16)$, and dermatomyositis $(n=4)$. Diagnoses in the remaining group included malignancies $(n=7)$, fibromyalgia $(n=2)$, spondyloarthropathy $(n=2)$, polymyalgia rheumatica $(n=1)$, polyneuritis $(n=1)$, sensible hemisyndrome $(n=1)$, mother of a child with neonatal lupus $(n=1)$, lymphocytic infiltration of the skin $(n=1)$, and hepatitis $C$ virus infection $(\mathrm{n}=1)$.

Table 1 gives the distribution of diagnoses according to the detailed anti-SSA/anti-SSB reactivity defined by line immunoassay with recombinant Ro52, recombinant SSB, and natural Ro60. Single anti-Ro52 reactivity increased the probability for systemic sclerosis $(34.2 \%, 95 \%$ CI $19.6-51.4$ versus $8.8 \%$ in the total anti-SSA and/or anti-SSB positive population) whereas the chances for SLE $(15.8 \%, 95 \%$ CI 6.0 to 31.3$)$, PM/DM ( $10.5 \%, 95 \%$ CI 2.9 to 24.8$)$ and pSS $(7.9 \%, 95 \%$ CI 1.7 to 21.4$)$ became comparable. Single reactivity towards Ro60 or anti-Ro60 combined with anti-Ro52 increased the probability for SLE (respectively $80.0 \%, 95 \%$ CI 44.4 to 97.5 and $52.2 \%$, $95 \%$ CI 30.6 to 73.2 ). Triple reactivity towards Ro52, Ro60 and SSB predisposed primarily to SLE $(55.8 \%, 95 \%$ CI 44.7 to $66.5)$, followed by pSS $(20.9 \%, 95 \%$ CI 12.9 to 31.1$)$. On the other hand, triple reactivity was significantly less indicative for $\mathrm{Scl}(1.2 \%, 95 \%$ CI 0.03 to 6.3$)$, CLE $(5.8 \%, 95 \%$ CI 1.9 to $13.1)$, and RA $(5.8 \%, 95 \%$ CI 1.9 to 13.1$)$.

Table 2 gives the distribution of diagnoses according to the immunodiffusion result. A positive result for anti-SSA on immunodiffusion, independent of the result for anti-SSB reactivity, strongly predisposed to SLE $(66.7 \%, 95 \%$ CI 46.0 to 83.5 and $61.4 \%, 95 \%$ CI 49.0 to 72.8 ) and to a significantly smaller extent to pSS $(18.5 \%, 95 \%$ CI 6.3 to 38.1 and $15.7 \%$, $95 \%$ CI 8.1 to 26.4$)$. Isolated anti-SSB immunodiffusion reactivity instead decreased the chances for SLE $(14.3 \%, 95 \%$ CI 3.0 to 36.3$)$ whereas in this subgroup, CLE $(23.8 \%$, 95\% CI 8.2 to 47.2$)$ and pSS $(33 \%, 95 \%$ CI 14.6 to 57.0$)$ became more probable. Sixty three of the 181 patients $(34.8 \%)$ were solely identified by line immunoassay and not by immunodiffusion. Negative results for immunodiffusion in the line immunoassay positive group were less indicative for pSS (4.8\%, 95\% CI 1.0 to 13.3$)$ whereas SLE $(28.6 \%, 95 \%$ CI 17.9 to 41.3$)$ and Scl $(23.8 \%, 95 \%$ CI 14.0 to 36.2$)$ were found in almost equal percentages.

Combining immunodiffusion and line immunoassay had only additional diagnostic value in the case of combined reactivity towards Ro52, Ro60, and SSB (table 3). A positive test result for anti-SSA on immunodiffusion in this group gave a probability for SLE of $69.2 \%$ (95\% CI 48.2 to 85.7 ) and $64.1 \%$ (95\% CI 47.2 to 78.8 ) and a significantly lower chance for pSS ( $19.2 \%, 95 \%$ CI 6.6 to 39.4 and $15.4 \%, 95 \%$ CI 5.9 to 30.5 ). On the contrary, the finding of a precipitin line on immunodiffusion for SSB but not for SSA in this profile decreased the probability for SLE ( $13.3 \%, 95 \%$ CI 1.7 to 40.5 ) whereas in that case there was a tendency towards an increased chance for pSS $(46.7 \%, 95 \%$ CI 21.3 to 73.4 ; table 3$)$.

\section{DISCUSSION}

The description of a large, consecutively identified cohort of anti-SSA and/or anti-SSB positive serum samples in the routine setting of a rheumatology laboratory offers the opportunity to look at a realistic representation of the diagnostic range associated with this type of autoreactivity. Most of the work on the value of autoantibodies has been carried out by 
testing samples from selected patients with well defined clinical disease..$^{4017}$ By contrast, by looking at sensitivity and specificity of autoantibody markers such as anti-SSA and anti-SSB, the current study rather provides clues for estimating the probability for a certain diagnosis given the anti-SSA/ anti-SSB status, taking into account that the a priori probabilities can differ according to the type of clinical practice and the specialty of the doctor ordering the test. Serum samples in our laboratory had a mixed origin, with about one third of the ANA positive samples coming from the rheumatology department. A positive ANA result itself has only weak predictive value for diagnosing SLE or other connective tissue diseases, even in a group whose serum samples are specifically referred for ANA testing. ${ }^{18}{ }^{19}$ Identification of more specific antinuclear reactivities significantly increases the predictive diagnostic value up to a level that is of real diagnostic value in specialist practice.

Anti-SSA and/or anti-SSB reactivity were identified in $11.8 \%$ of the ANA positive patients. ${ }^{1}$ The most prevalent disease associated with anti-SSA/SSB autoreactivity, was SLE. Especially, the combined triple reactivity (anti-Ro52, antiRo60, and anti-SSB) and anti-Ro60 with or without anti-Ro52 reactivity makes this diagnosis highly probable. Our data confirm that anti-Ro60 reactivity without anti-Ro52 and anti-SSB reactivity is very indicative for SLE. ${ }^{20}$ By contrast, none of the 26 patients with pSS had only antibodies to Ro60, whereas anti-Ro52 reactivity was present in 25 of the 26 patients. Previous evidence has been presented that the major anti-SSA response consists of anti-Ro52 antibodies in $\mathrm{pSS}^{4}$ and anti-Ro60 antibodies in SLE. ${ }^{17}$ Patients with Scl or DM rarely present with combined anti-Ro52, anti-Ro60, or anti-SSB antibodies. Our present study, representing a consecutive series of samples, suggests that the finding of an isolated response to Ro52 predisposes most for systemic sclerosis and almost equally for SLE and DM. All patients diagnosed with DM were also encountered in this serotype group. The phenomenon of anti-Ro52 antibodies in DM/PM and Scl, without concomitant anti-Ro60 and anti-SSB antibodies, has been described previously. ${ }^{21-23}$ One study also found a strong association between anti-Ro52 and anti-Jol antibodies. ${ }^{21}{ }^{23}$ Two of our patients with DM had anti-Jol reactivity. None of the patients with DM and only one patient with scleroderma were identified by immunodiffusion.

To our knowledge, no other studies examined the diagnostic range associated with the detailed anti-SSA/anti-SSB serology in a large consecutive ANA-positive cohort. This analysis underscores the interest in identifying the detailed reactivity of anti-SSA/SSB autoantibodies, as this alters the ratios of associated diagnoses, and thus the diagnostic probabilities. Evidence has been provided that patients with undifferentiated connective tissue disease and antibodies to SSA can progress in a relatively short period to well defined connective tissue diseases. ${ }^{24}$ The possibility exists that some of our patients classified as "other" will evolve to defined connective tissue diseases over time.

It seemed that immunodiffusion had somewhat higher diagnostic value than line immunoassay (table 2). However, 63 serum samples positive on line immunoassay were not identified by immunodiffusion versus two serum samples that were solely retrieved by immunodiffusion. Most of these 63 patients had a defined connective tissue disease. The higher sensitivity of the line immunoassay could mainly be attributed to the earlier described better performance of this assay in detecting anti-Ro52 and anti-SSB antibodies. ${ }^{25}$ Based on this higher detection level and on the fact that the diagnostic range associated with anti-SSA/anti-SSB reactivity differs significantly according to the fine serotype, we suggest screening for anti-SSA/anti-SSB reactivity by line immunoassay. When confronted with triple reactivity to Ro52, Ro60, and SSB on line immunoassay, we found that additionally performed immunodiffusion discriminates between SLE and pSS. An additional advantage of the line immunoassay technique in clinical practice is that with one test result information can be obtained on the range of simultaneous occurrence of autoantibodies in connective tissue disease. Besides anti-Ro52 and anti-Ro60, this assay also detects autoreactivity towards the different antigenic determinants of the RNP-antigen (RNP-A, C, and 70) and the Sm antigen (SmB and SmD). ${ }^{25}$ A major challenge for autoantibodies in general and for anti-SSA/anti-SSB in particular is now to find out whether reactivities to subtypes of antigens orientate towards a specific diagnosis or a specific feature common to different clinical entities, as well as to understand which mechanisms induce these different reaction patterns in autoimmune patients.

\section{Authors' affiliations}

I Peene, E M Veys, F De Keyser, Department of Rheumatology, Ghent University Hospital, Ghent, Belgium

L Meheus, Innogenetics, Ghent, Belgium

\section{REFERENCES}

1 Peene I, Meheus L, Veys E, De Keyser F. Detection and identification of antinuclear antibodies (ANA) in a large and consecutive cohort of sera referred for ANA testing. Ann Rheum Dis 2001;60:1131-6.

2 Ben-Chetrit E. The molecular basis of the SSA/Ro antigens and the clinical significance of their autoantibodies. $\mathrm{Br} J$ Rheumatol 1993:32:396-402

3 Tzioufas AG, Moutsopoulos HM. Clinical significance of autoantibodies to Ro/SSA and La/SSB. In: Van Venrooij WJ, Maini RN, eds. Manual of biological markers of disease. Dordrecht, Kluwer Acadamic 1996;C4.1:1-14

4 Lopez-Longo FJ, Rodriguez-Mahou M, Escalona M, Perez T, Monteagado I, Sanchez-Atrio A, et al. Heterogeneity of the anti-Ro (SSA) response in rheumatic diseases. J Rheumatol 1994;21:1450-6.

5 Brucato A, Buyon J, Horsfall A, Lee L, Reichlin M. Fourth International Workshop on neonatal lupus syndromes and the Ro/SSA-La/SSB system. Clin Exp Rheum 1999;17:130-6.

6 Kelekar A, Saitta MR, Keene JD. Molecular composition of Ro small ribonucleoprotein complexes in human cells. Intracellular localization of the 60- and 52-kD proteins. J Clin Invest 1994;93:1637-44.

7 Boire G, Gendron M, Monast N, Bastin B, Ménard HA. Purification of antigenically intact Ro ribonucleoproteins: biochemical and immunological evidence that the $52-\mathrm{kD}$ protein is not a Ro protein. Clin Exp Immunol 1995; 100:489-98

8 Scofield RH, Farris AD, Horsfall AC, Harley JB. Fine specificity of the autoimmune response to the Ro/SSA and La/SSB ribonucleoproteins. Arthritis Rheum 1999:42:199-209.

9 Tan EM, Smolen JS, MC Dougal JS, Butcher BT, Conn D, Dawkins R. et al. A critical evaluation of enzyme immunoassays for detection of antinuclear autoantibodies of defined specificities. I Precision, sensitivity and specificity. Arthritis Rheum 1999;42:455-64.

10 Zimmerman C, Smolen JS, Graniger W, Petera P, Fabini G, Hassfeld $W$, et al. Fine specificity of anti-Ro(SSA) autoantibodies and clinical manifestations in patients with systemic lupus erythematosus. J Rheumatol 1996;23:1897-903.

11 McCauliffe DP, Wang L, Satoh M, Reeves WH, Small D. Recombinant $52 k$ Da Ro(SSA) ELISA detects autoantibodies in Siögren's syndrome that go undetected by conventional serologic assays. J Rheumatol 1997;24:860-6

12 Tan EM, Cohen AS, Fries JF, Masi AT, Mc Shane DJ, Rothfield NF, et al. The 1982 revised criteria for the classification of systemic lupus erythematosus. Arthritis Rheum 1982;25:1271-7.

13 Arnett FC, Edworthy SM, Bloch DA, Mc Shane DJ, Fries JF, Cooper NS, et al. The American Rheumatism Association 1987 revised criteria for the classification of rheumatoid arthritis. Arthritis Rheum 1988;31:315-24.

14 Masi AT, Rodnan GP, Medsger TA Jr, et al. Preliminary criteria for the classification of systemic sclerosis (scleroderma). Arthritis Rheum 1980;23:581-90.

15 Targoff IN, Miller FW, Medsger TA, Oddis CV. Classification criteria for the idiopathic inflammatory myopathies. Curr Opin Rheumatol 1997;9:527-35

16 Vitali C, Bombardieri S, Moutsoupoulos M, Balestrieri G, Bencivelli W, Bernstein RM, et al. Preliminary criteria for the classification of Siögren's syndrome: results of a prospective concerted action supported by the European Community. Arthritis Rheum 1993:36:340-7.

17 Ben-Chetrit E, Fox RI, Tan EM. Dissociation of immunoresponses to the SS-A (Ro) 52-kd and 60-kd polypeptides in systemic lupus erythematosus and Siögren's syndrome. Arthritis Rheum 1990;33:329-35.

18 Slater CA, Davis RB, Shmerling RH. Antinuclear antibody testing. A study of clinical utility. Arch Intern Med 1996;156:1421-5.

19 Emlen W, O'Neill L. Cinical significance of antinuclear antibodies. Arthritis Rheum 1997;40:1612-18. 
20 Slobbe RL, Pruijn GJM, Damen WGM, Van Der Kemp JWCM, van Venrooij WJ. Detection and occurrence of the 60-and 52-kD Ro (SS-A) antigens and of autoantibodies against these proteins. Clin Exp Immuno 1991;86:99-105.

21 Ruties SA, Vree Egberts WTM, Jongen P, Van Den Hoogen F, Pruijn GJM, van Venrooij WJ. Anti-Ro52 antibodies frequently co-occur with anti-Jo-1 antibodies in sera from patients with idiopathic inflammatory myopathy. Clin Exp Immunol 1997;109:32-40.

22 Frank MB, Mc Cubbin V, Trieu E, Wu Y, Isenberg DA, Targoff IN. The association of anti-Ro52 autoantibodies with myositis and scleroderma autoantibodies. J Autoimmun 1999;12:137-42.
23 Brouwer R, Hengstman GJ, Vree Egberts W, Ehrfeld H, Bozic B, Ghirardello $A$, et al. Autoantibody profiles in the sera of European patients with myositis. Ann Rheum Dis 2001;60:116-23.

24 Cavazzana I, Franceschini F, Belfiore N, Quinzanini M, Caporali R, Calzavara-Pinton $P$, et al. Undifferentiated connective tissue disease with antibodies to Ro/SSA: clinical features and follow up of 148 patients. Clin Exp Rheumatol 2001;19:403-39.

25 Meheus L, Van Venrooij WJ, Wiik A, Charles PJ, Tzioufas AG, Meyer $O$, et al. Determination of the fine specificity of antinuclear antibodies in connective tissue diseases using a multiparameter line immunoassay (LIA) based on recombinant proteins. Clin Exp Rheumatol 1999;17:205-14.

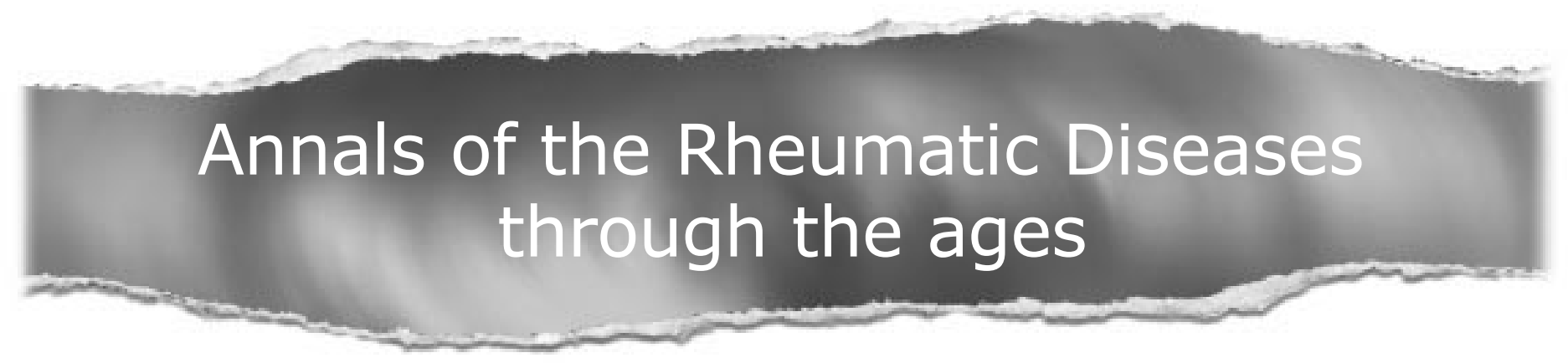

Browse the Archive

Annals of the Rheumatic Diseases online has an archive of content dating back to 1965.

Full text from 1997; abstracts from 1975; table of contents from 1965

www.annrheumdis.com 